\title{
Study of Radiation Therapy Treatment Effect in PAIN Management for Metastatic Breast Cancer in RICK
}

\author{
Lara Abdelmunim I Abdelrahman*, Mohamed E Gar Elanabi and Nahla gafer \\ College of Medical Radiological Science, Sudan
}

*Corresponding author: Lara Abdelmunim I Abdelrahman, College of Medical Radiological Science, Khartoum, Sudan

\begin{abstract}
Pain is one of the most common, unattended and unsolved problem for the cancer patients. Radiotherapy is successful, time efficient, well tolerated. The goal of palliative radiotherapy symptoms relief at the site of primary tumour or from metastic lesion. The study examined the association between Palliative radiotherapy and the improvement of the symptoms associated with metastatic breast cancer. These include metastatic in the spinal cord, bone and associated pain, also dealt with the connection between it and the improvement of the standard of life of the patient and toxic and other important factors and was achieved through a set of international standards questionnaire by which the calibration of the result. This study was conducted in the Center of tumours treatment in Sudan, represented in Khartoum oncology Hospital (RICK) in the period 2014 to 2017. The findings support the hypothesis that radiotherapy is an effective treatment for a proportion of patients with MBC related pain, with 35\% of assessable patients experiencing a clinically meaningful improvement in their pain. Of these, $12.5 \%$ had a complete improvement in their pain. There were no specific features that differentiated the complete responders from the other number of patients though this may be due to the small number of complete responders.
\end{abstract}

Keywords: Palliative Radiotherapy; Pain Management; Metastic breast cancer; Pain response

\section{Introduction}

Metastatic breast cancer (MBC) is incurable, but still treatable, especially if there are limited metastases. The intent of treatment is palliative, providing symptomatic relief and optimization of the length and quality of life. Median survival is approximately 18 to 24 months in these patients. About $34-50 \%$ of patients receiving radiotherapy are of palliative intent Janjan N [1]. Similar to other clinical domains, the practice of palliative radiotherapy is always guided by basic ethical principles and available clinical evidence. It requires sophisticated assessment to balance the potential benefits and burdens to the patients with respect to patient's autonomy and expectations, and consideration of logistical factors Palliative radiotherapy is mainly indicated to relieve various local symptoms in cancer patients; to prevent debilitation such as spinal cord compression and pathological fracture; and to achieve durable loco regional control (Wong K2004) in Table 1. The effectiveness has been confirmed by cumulative clinical evidence. For metastatic bone pain, palliative radiotherapy can achieve an overall pain response rate of 59-62\%, and a complete pain response rate of 32$34 \%$. For multiple brain metastases, the overall response rate to external irradiation is around 60\% with $30-40 \%$ achieving marked neurological improvement (Figure 1).

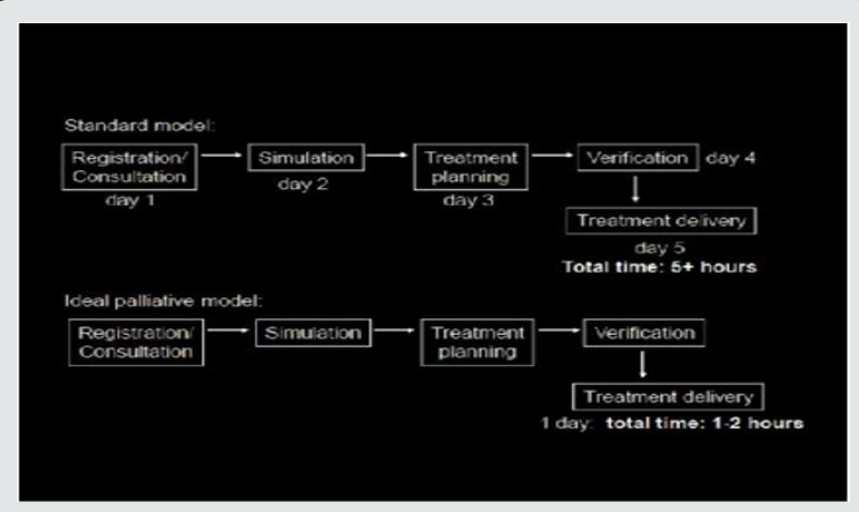

Figure 1: Schematic block diagram demonstrating the multiple steps in the radiation treatment Process. 
Table 1: Indication of palliative radiotherapy.

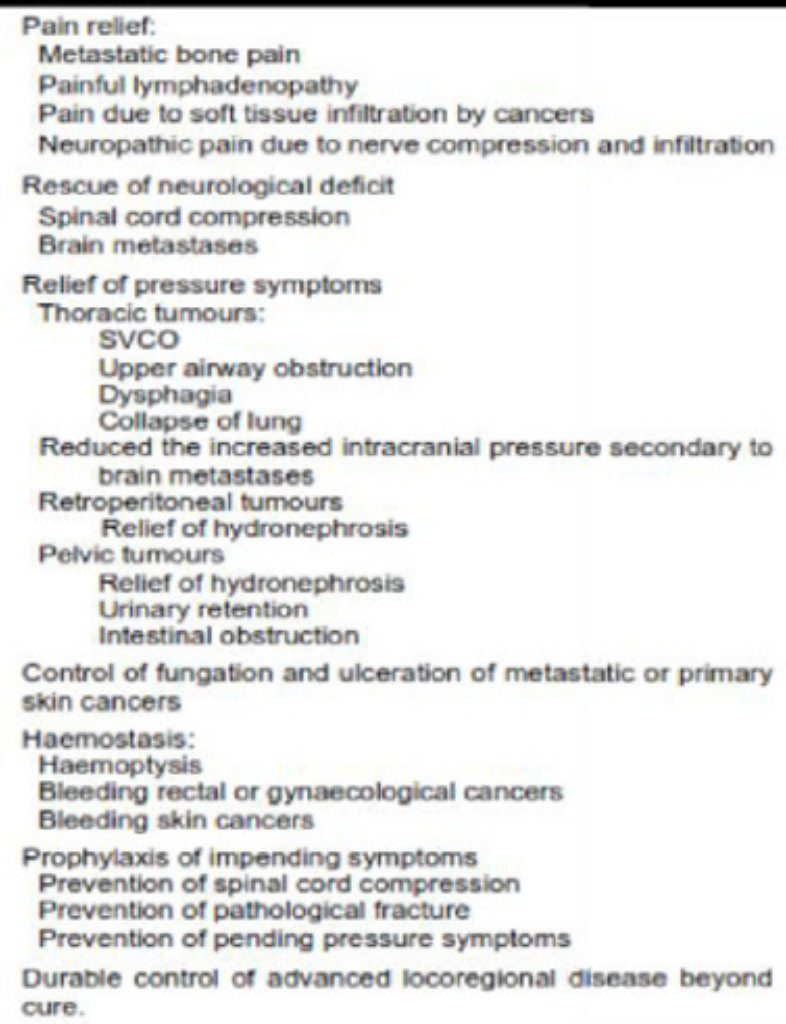

\section{Principle of Palliative Radiotherapy}

The intention of giving radiotherapy for palliation of symptoms is improvement in quality of life by decreasing or eradicating symptoms. This will not be achieved if the treatment itself induces a lot of side effects. Also, patients with metastatic cancer have a reduced life span, this may only be months, and therefore the treatment itself should not consume a major portion of the patients remaining lifespan. The major benefit of radiotherapy is the speed with which symptom improvement develops and the certainty of response. Sufficient radiation dose must be given to ensure that the symptom response will last for the rest of the patient's life. Too low a dose means retreatment at some later time is needed. Hence guiding principles are:

a. Accurate anatomical localization of the symptomatic tumour deposit.

b. Simple treatment techniques and field arrangements

c. Short hypo fractionated treatment regimes.

d. Moderate dose treatment to achieve a good predictable response and to keep treatment toxicity to a minimum.

e. Consider the patient's over all life expectancy when determining the treatment aims and the treatment duration (Table 2).
Table 2.

\begin{tabular}{|c|c|}
\hline Site & Patient position \\
\hline Head & Supine, prone for posterior le sions, head rest \\
\hline Spine: & $\begin{array}{c}\text { Prone if patient able (otherwise supine), head } \\
\text { rest }\end{array}$ \\
\hline Pelvis & Supine \\
\hline Extremities & $\begin{array}{c}\text { Treatment site to be possitioned away from } \\
\text { normal tissue }\end{array}$ \\
\hline Scapula & Supine unless mark-up on-set \\
\hline Ribs & $\begin{array}{c}\text { Dependent on site of ribb metastasis to be } \\
\text { treated }\end{array}$ \\
\hline Sternum & Supine \\
\hline
\end{tabular}

\section{Radiotherapy for the Treatment of MBC}

Palliative radiation therapy for metastatic breast cancer can generally be performed with simple techniques and simple technology. The radiation treatment process is complex and consists of multiple steps. These are broadly summarized in a simplified form in Figure 2. The steps are not always necessarily in the same order nor are all the steps always needed. The latter is especially true for palliative radiation therapy where CT scanning and target volume delineation are not always required, particularly when a large field are used to treat systemic disease or pain.

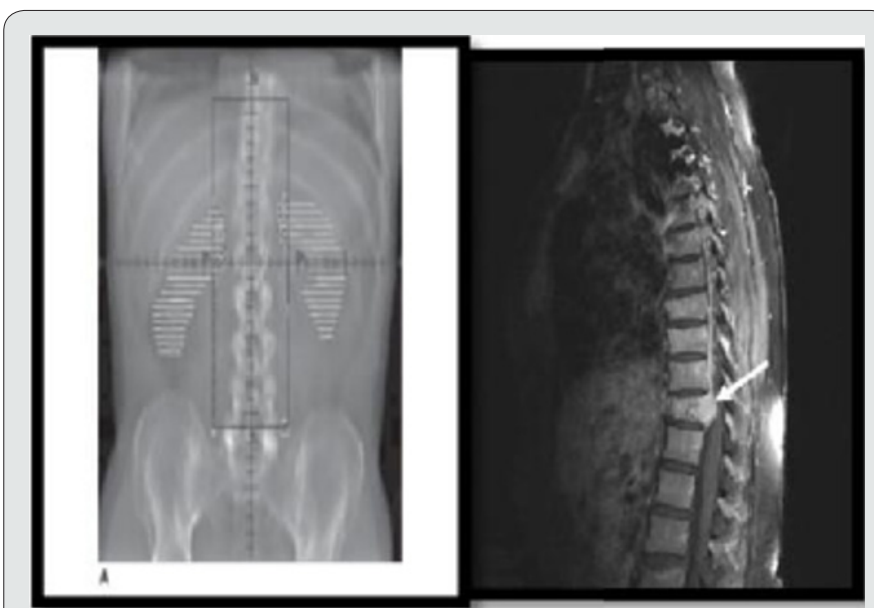

Figure 2: Field Arrangement in MSC.

\section{The Role of Palliative RT in Metastatic Breast Cancer MBC}

\section{Bone Pain and Bone Metastasis}

The skeleton is one of the commonest sites for metastatic cancer of any type. Whilst cancer in the bones is not usually directly life threatening it is frequently a source of pain which is a major debility. On occasions the more disabling complication of pathological fracture, spinal cord com pression and hypocalcaemia may also occur. Local irradiation of one or more painful bone deposits is associated with a high probability of pain relief. However, other studies show that longer term pain relief, greater 
tumour shrink age, and thus, fewer episodes of retreatment are achieved by a multifaction treatment program. Hence the choice of dose and fraction number needs to be tailored to the patient's general condition, expected survival and convenience of access. While single dose treatment may be adequate for pain relief, when tumour shrinkage is the goal this may not be adequate. For example, in spinal cord compression, where extension of soft tissue tumour from the vertebral bone into the spinal canal causing the spinal cord to be compressed and neurological impairment, or in a weight bearing bone, where sufficient bone destruction has occurred to reduce the mechanical strength of the $b$ one. In these situations, significant tumour shrinkage is required to relieve symptoms. So, short course fractionated treatment is preferred either 30Gy in 10 factions or 20Gyin 5 fractions at 5 fractions per weeks (Table 3).

Table 3: Indication of palliative radiotherapy.

\begin{tabular}{|cc|}
\hline LANSS & No of Patient \\
\hline LANSS Score $>12$ & 53 \\
LANSS Score $<12$ & 33 \\
\hline
\end{tabular}

\section{Treatment planning of MBC in spinal Cord compression:}

It occurs in three sites:

$\begin{array}{ll}\text { Thoracic spine } & 60 \% \\ \text { Lumbosacral spine } & 30 \% \\ \text { Cervical spine } & 10 \%\end{array}$

a. Immobilization: Body cushion with comfortable prone head rest.

b. Field Arrangement: Prescribe at $5-8 \mathrm{~cm}$ depending on particular level in cord (cervical - lumbar)

i. Laterally

$1 \mathrm{~cm}$ margin beyond the pedicle to cover the spinal cord and meninges along the nerve root up to the spiral ganglia.

ii. Caudal

$1 \mathrm{~cm}$ below the termination of the sac L5-S3.

Technique used to Treat MSCC:
a. SSD
b. gantry angle $=0$

Dose:

Treat extradural disease-visualize on MRI +2 vertebral bodies
a. $8 \mathrm{~Gy} / 1 \mathrm{~F}$
b. 20Gy / 5F / 5 days
c. 30Gy / 10F / 2 weeks

\section{Indicated of radiotherapy treatment of bone irradiation:}

a. Bone metastases presenting with pain not adequately controlled by analgesia.

b. Bone metastases causing mass effect.

c. Inoperable impending / existing pathological fracture.

d. Pathological fracture following surgical fixation.

\section{Immobilization}
a) Head / C-Spine
b) Thermoplastic Shell
c) Extremities: may benefit from Body foam

\section{Target Definition}

The GTV = the volume of metastatic disease, as determined by diagnostic imaging and clinical examination. The CTV = The GTV + surrounding bone at risk of microscopic involvement. The PTV = The CTV with a margin dependent on the treatment site Field borders should cover the area of metastatic involvement (the CTV) with a $1-3 \mathrm{~cm}$ margin while making anatomical considerations to aid future matching of fields and to avoid treatment of normal tissues. For post-operative treatment, the field should include metal work with a $12 \mathrm{~cm}$ margin (Figure 3 ).

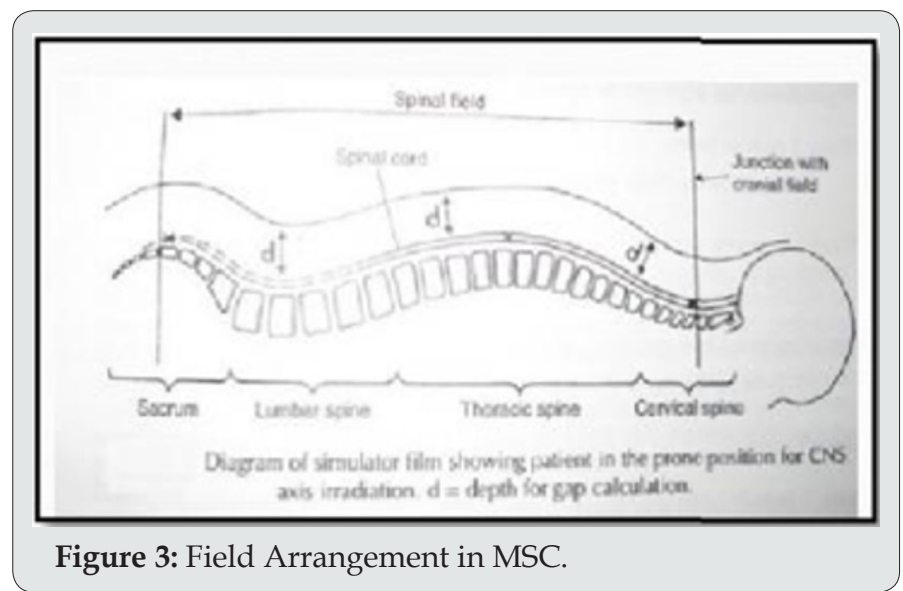

\section{Prescription Dose}

I. 6Gy/1F: Upper hemi body irradiation

II. 8Gy/1F: Standard dose for palliation of painful bone metastic

III. 20Gy/5F: May be considered instead of single fraction for pat with a very good performance status with:
a. Inoperable impending pathological fracture
b. Established pathological fracture
c. Prior surgical fixation 
d. Re irradiation of bone metastases

e. Large field size with a significant volume of normal tissue

I. 30Gy/10F: May be considered for patients with a good performance status and good prognosis (e.g. solitary bone metastasis from breast carcinoma).

II. 25Gy/5F: Radio biologically equivalent to $30 \mathrm{~Gy}$ in $10 \mathrm{~F}$. May be considered for patients with a good performance status and good prognosis with a tumour with a high fraction sensitivity / low a/ß ratio (Whelan TJet 2010).

\section{Materials and Methods}

The study was conducted at Khartoum oncology hospital (RICK). The study conducted from 2014 to 2017. The rationale for this study is to development tools to assessment and evaluation patient with late stage breast cancer in symptoms control by using symptoms improvement ratio (SIR) to evaluate benefit of RT in palliative cases, what is mean by this is that now, in principle it is possible to evaluate the radiotherapy protocol by using simple and fast tools such as questionnaire, clinical fellow up. The patients were planning and treatment by a fluoroscopy of patient by using simple planning with two image AP/PA -LAT view of patients to detect the patient separation the images were performed using two type of machine (Hustise Castter unit, Terasix UPJ machine) the patients were planning depended on area of metastic. Some cases were planning manual depending on previous patients images and anatomical land mark. The patient were treat in both cobalt 60 unit or linear accelerator unit. It is analytical study where the data collected prospectively. which was amongst the metastatic breast cancer patients in Sudan. Study of radiotherapy regimes for the treatment of in patients with MBC. A study schema is shown in Figure 4.

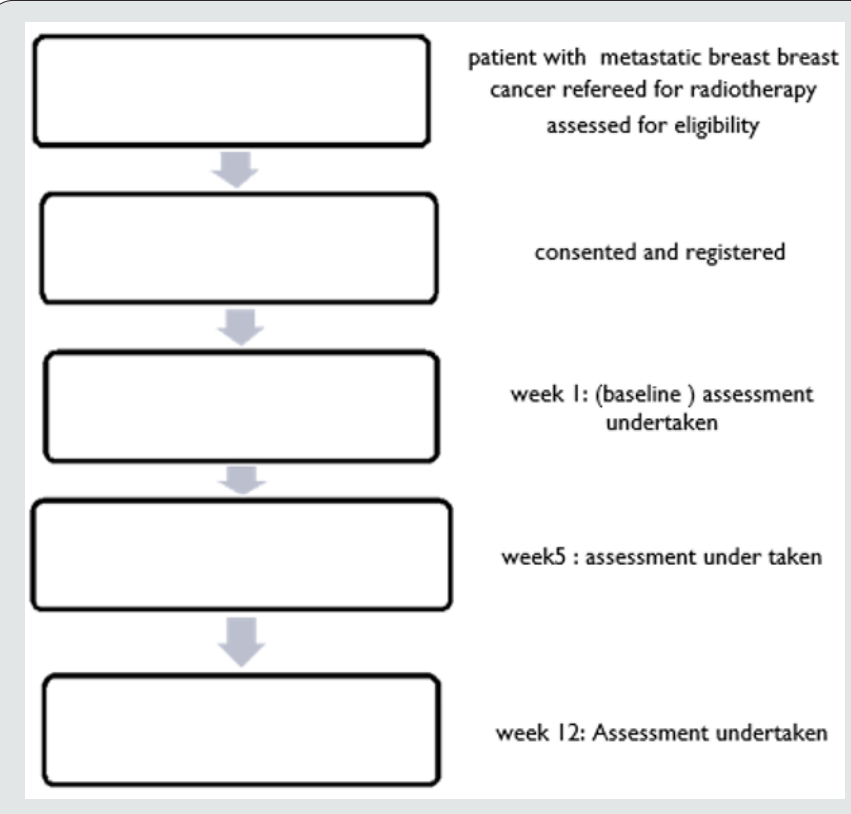

Figure 4: Shows the individua 1 components of the BPI.

\section{Pre-Study Assessment}

One of the challenges of conducting a study examining a pain intervention in patients with advanced cancer is that changes in analgesia may occur during the study period. In such cases, it becomes very difficult to disentangle any improvements in pain as a result of the study intervention from any changes in concomitant analgesia. One of the ways this can be addressed is by stabilizing pain and analgesia prior to study entry. This approach was adopted for potential Patients in this study. Prior to study consent, patients were reviewed by Dr. Nahla gaffer and Dr. Mohaga (Palliative Medicine), often with multiple visits or telephone consultations over several weeks, enabling background analgesia to be optimized and pain stabilized, where possible, before study entry. This also resulted in some patients' analgesia improving to the extent that their pain was no longer severe enough for study entry. Following optimization of analgesics, if patients were eligible, written informed consent was obtained. Baseline visit at the baseline visit; an assessment of performance status was made along with a physical examination. All previous treatments for Metastic breast cancer MBC-surgery, chemotherapy or radiotherapy - were documented along with the medication history which listed all medicines taken in the previous 24 hours. Baseline toxicity assessment was performed, and all study questionnaires were completed (Table 4). In order to improve compliance, rather than leaving the patient to complete the questionnaires, a researcher completed the questionnaires based on the answers received from the patient. Take blood samples from patients at baseline and week 12. The aims of these blood tests were twofold. The first aim was to explore the possibility that proteins could be identified which might help to predict a response to radiotherapy. The second aim was to explore the possibility of toxicity due to radiotherapy.

Table 4: LANSS score answer questionnaire.

\begin{tabular}{|c|c|c|c|c|}
\hline Variable & $\begin{array}{c}\text { Average } \\
\text { lowest BPI } \\
\text { Score }\end{array}$ & $\begin{array}{l}\text { High base } \\
\text { line answer }\end{array}$ & $\begin{array}{c}\text { Low base } \\
\text { line answer }\end{array}$ & $\begin{array}{c}\text { Average } \\
\text { higher BPI } \\
\text { score }\end{array}$ \\
\hline worst & 6 & 10 & 0 & 8 \\
\hline least & 0.5 & 10 & 0 & 3 \\
\hline average & 4 & 10 & 1 & 6 \\
\hline right now & 1 & 10 & 0 & 4 \\
\hline $\begin{array}{l}\text { general } \\
\text { activity }\end{array}$ & 5 & 10 & 0 & 8 \\
\hline mood & 3 & 10 & 0 & 5 \\
\hline $\begin{array}{c}\text { walking } \\
\text { ability }\end{array}$ & 3 & 10 & 0 & 7 \\
\hline work & 4 & 10 & 0 & 8 \\
\hline relations & 0.5 & 10 & 0 & 6 \\
\hline sleep & 0.5 & 10 & 0 & 8 \\
\hline enjoyment & 4 & 10 & 0 & 8 \\
\hline
\end{tabular}




\section{Week 1 visit}

Patients were seen eight (+/-three) days after the start of radiotherapy for their week 1 visit. At this consultation, current medication was recorded, including analgesics in the past 24 hours. Any toxicity from radiotherapy was documented and the questionnaires were repeated. Current symptoms were documented following the week 1 visit, patients received weekly phone calls in order to monitor symptoms and assess analgesic requirements.

\section{Week 5 visit}

Patients were seen 35 (+/-5) days from the start of radiotherapy. At this visit, all the study visits performed at week 1 were repeated. In addition. However, if patients were unable to attend, efforts were made to see them at home. After the week 5 visit, the weekly phone calls continued until the week 12 visit.

\section{Week 12 visit}

At the week 12 visit, all assessments undertaken at week 5 were repeated. Following this visit, patients were discharged back to their local oncology teams and study involvement ceased. During the course of the study, if patients' analgesia required to be altered, this was done as per usual clinical practice.

\section{Questionnaires: Brief Pain Inventory (BPI)}

The BPI is a multi-dimensional pain assessment tool. It was designed to serve two purposes; to measure the intensity of pain and to assess the level of interference of pain on daily function. It was developed for use in cancer patients and has been extensively validated in both cancer and non-cancer patients (Cleeland, [2]. Portenoy RK [3]. All questions in the BPI relate to the previous 24 hours. The section on pain intensity asks the worst, least and average pain as well as the pain right now. Subjects are asked to score each answer from $0-10$ where 0 is "no pain" and 10 is "pain as bad as you can imagine". It also asks the participant to rate the percentage of pain relief they experience from whatever pain treatments or medications they are currently on, ranging from $0-100$. The second section of the BPI focuses on the level of interference of pain on the subject's lifestyle, namely their general activity, mood, walking ability, normal work, relations with other people, sleep and enjoyment of life. Again, the scores are from 0-10 with 0 corresponding to "does not interfere" and 10 representing "completely interferes" with each question that has been asked. Once the questionnaire has been completed, the total BPI score can be calculated and repeated to assess the impact of an intervention on the subject's pain. For the study, the total score at baseline was calculated. A pain response was taken as a30\% drop in BPI score from the baseline assessment Portenoy R [3].

\section{Short form McGill Pain Question}

(SF-MPQ) The MPG is a scale for assessing pain using verbal descriptors. It was designed to allow patients to express the intensity and quality of their pain Melaka, R [4]. A short form version was developed in 1987 Melzack [5]. In 2009, this was further modified in order to develop a single measure for both neuropathic and non-neuropathic pain, Dworkin [6]. Amongst other purposes, it was planned that this questionnaire, SF-MPQ2, could be used in treatment response studies lead assessment of Neuropathic symptoms and sign (LANSS) The LANSS was developed in 2001 as a tool to identify patients who are likely to have neuropathic pain Bennett [7]. It has been extensively validated Kaki [8] (Yucel, A 2004). The assessment consists of two sections; a pain questionnaire and sensory testing. In the pain questionnaire, subjects are asked five yes/no questions concerning their pain. With the sensory testing, the subject is examined for alloying and for altered pin-prick threshold. Combining the scores for the questionnaire and the sensory testing gives a maximum score of 24. A score of $>12$ suggests that neuropathic mechanisms are likely to be contributing to the patient's pain, whereas a score of $<12$ suggests that neuropathic mechanisms are unlikely to be contributing to the patient's pain.

\section{Quality of Life Questionnaire (EORTC QLQ-C30)}

The EORTC QLQ-C30 is a validated questionnaire designed to assess the quality of life of cancer patients (Aaronson [9]. It incorporates nine multi-item scales: five functional scales (physical, role, cognitive, emotional and social); three symptom scales (fatigue, pain, and nausea and vomiting); and a global health and quality-of-life scale. As can be seen in the paragraphs above, with the exception of the NRS for night sweats, all of the questionnaires used in the study have been validated. The first step in the validation process involves a questionnaire being reposed [10].

\section{Study Endpoints}

\section{Primary}

As the primary aim of the prospective study was to examine if radiotherapy is an effective treatment for $\mathrm{MBC}$, the primary endpoint was to assess if there was a clinically significant improvement in pain 5 weeks following radiotherapy. A clinically significant improvement in pain was defined as a $>30 \%$ reduction from baseline in total BPI score Portenoy, R.K [3].

\section{Secondary}

There were a large number of secondary endpoints, all of which were exploratory in nature. The rationale for this was to inform future randomized studies examining radiotherapy in MBC. Endpoints were assessed at weeks 1, 5\&12 weeks post radiotherapy with the primary analysis at week 5 , unless otherwise stated.

\section{Secondary endpoints were as follows}

To examine the effect of radiotherapy on pain at weeks 1 and 12 post radiotherapy, assessed using the BPI. A clinically significant improvement in pain was defined as a $>30 \%$ reduction from baseline in total BPI score (Cleeland CS [2]. To examine the 
effect of radiotherapy on quality of life using the EORTC QLQ-C30 questionnaire (version 3.0 ) including lung cancer module EORTC QLQ-LC13 [11].

\section{The Results}

At weeks 1 and 12, the pain response rate was 27.5\% (CI14-6\%43.9\%) and $15.0 \%$ (CI5.7\%-29.8\%) respectively, on an intention to treat analysis. Based on complete case analysis, the proportion of pain responders at week 1 was $36.7 \%$ (CI19.9\%-56.1\%) and at w eek 12 was $33.3 \%$ (CI13.3\%-59.0). Although

32patients completed the week 1 Assessment, two of them ha d incomplete data and so were not evaluable. Eighteen patients were evaluable at week 12 .

\section{Pain Characteristics}

The sensory component of the SF-MPQ is shown in Figures 4-6. The words most commonly chosen to describe the pain were aching, tender and sharp being reported by 32 (86.5\%), 29(78.4\%) and $27(73 \%)$ of patients respectively [12]. The median (IQR) for average pain and worst pain was 4 (4-6) and 8 (6-8) respectively. General activity, normal work and enjoyment of life scored the highest on the interference scores. Relationships appeared to be relatively unaffected by the pain. Fifty -three patients, $31.4 \%$, had a total LANSS >12 while $33(68.6 \%)$ patients, had a LANSS < 12. An analysis was performed to assess whether there was an $y$ association between total LANSS, BPI and MPQ. The median total BPI for patients with a LANSS <12 was 52 (IQR 41.00 - 59.50) versus 69.50 (IQR $61.00-84.00$ ) for patients with a LANSS $>12$, $\mathrm{p}=0.004$. Similarly, comparing the LANSS with t he SF-MPQ, the median SF-MPQ for those with a LANSS $<12$ was 10.00 (IQR 8.00 13. 50) versus 15.00 (IQR $12.00-18.00$ ) for patients with a LANSS $>12, \mathrm{p}=0.012$ (Figures 7-9).

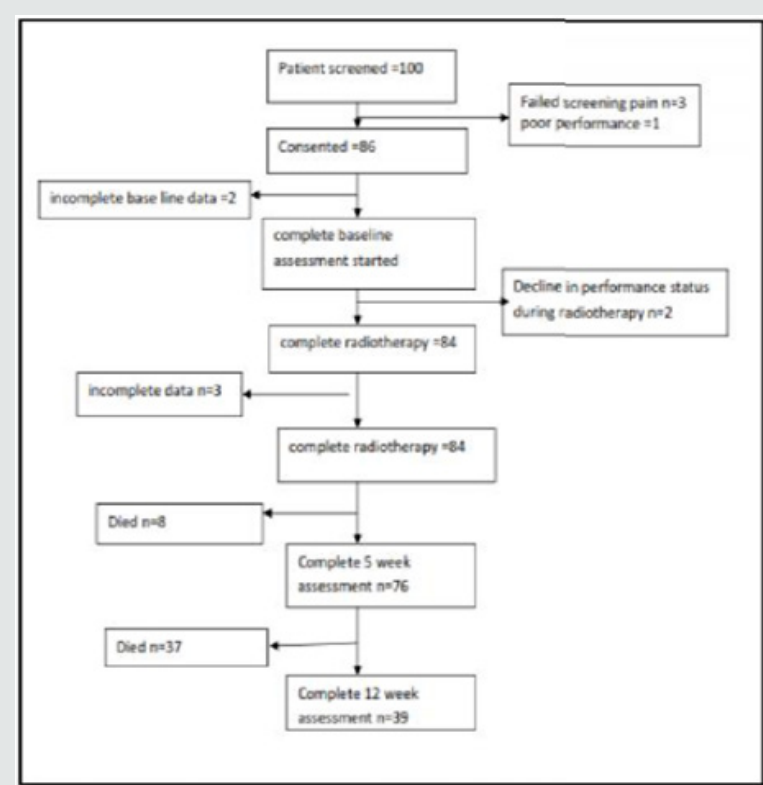

Figure 5: Patients disappoint.

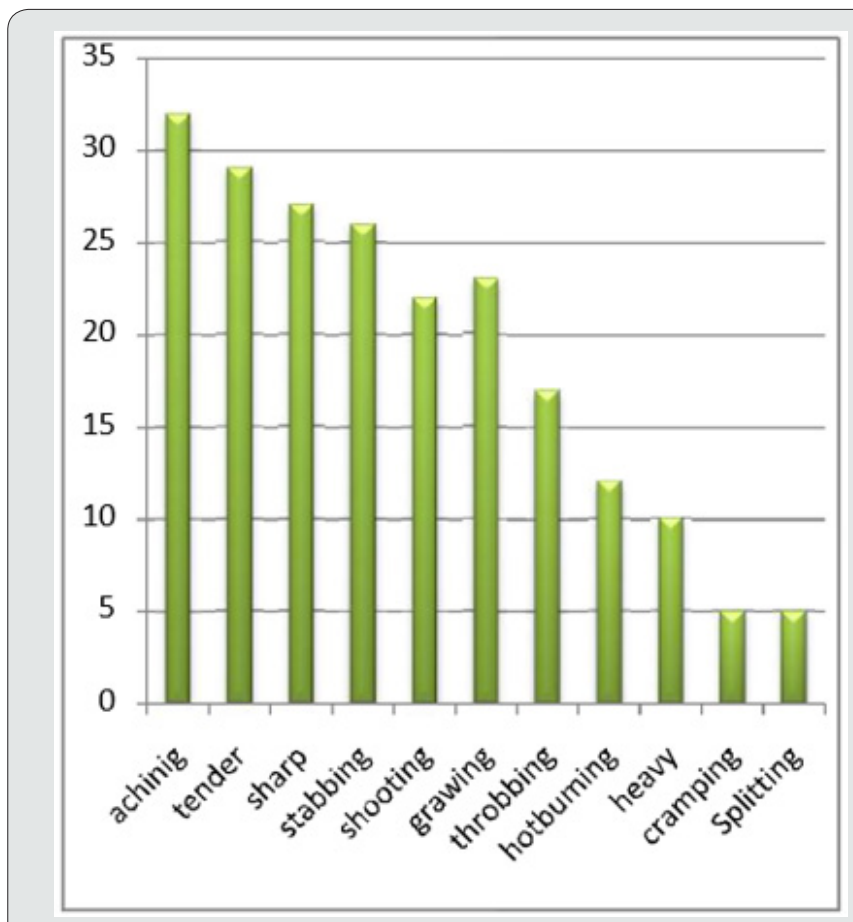

Figure 6: 4SF-M PQ.

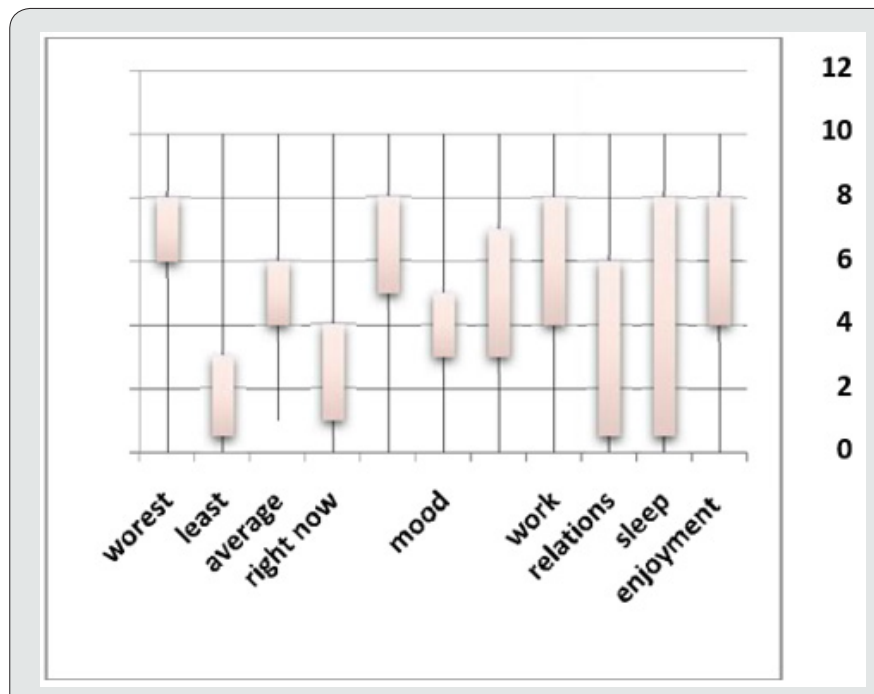

Figure 7: BPI Box plots of visual analogue components from baseline BPI questionnaire.

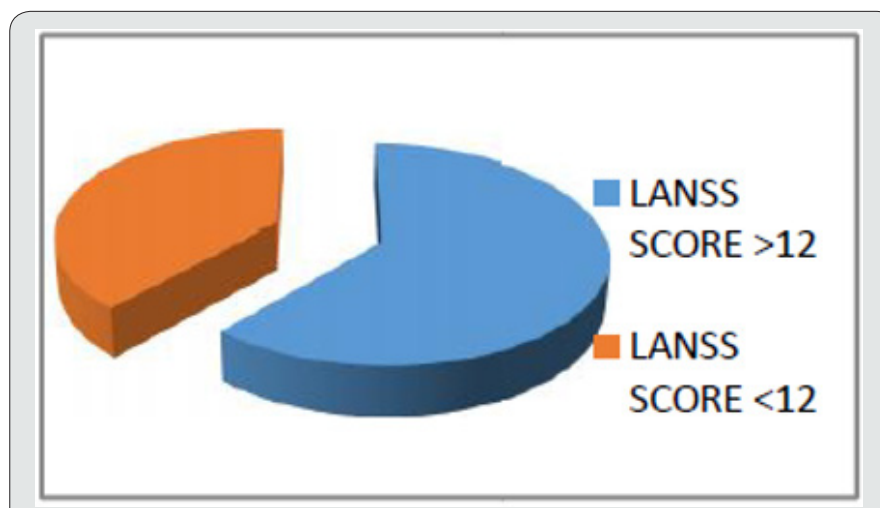

Figure 8: Pie graph of LANSS score answer Questionnaire. 


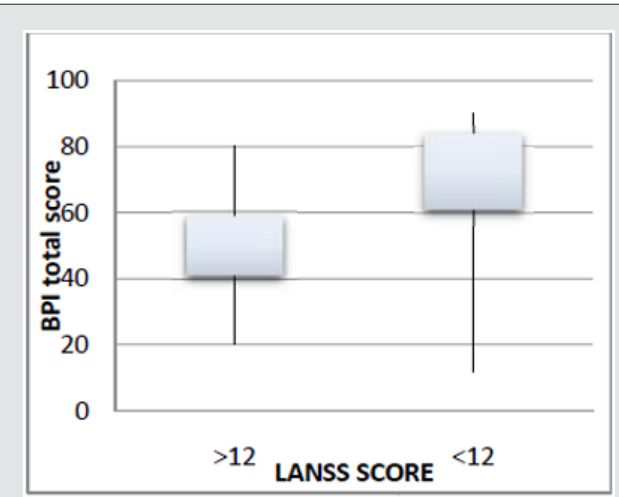

Figure 9: BPI with LANSS.

\section{Conclusion}

The study data was retrieved from patients' files system of Radiation and Isotopes Center of Khartoum (RICK) which included patient age, grade, type of treatment, type of cancer cells and site of tumor MITS. They were followed for 12 weeks. As can be seen from the short median survival of patients in the study, this is a frail population with poor life expectancy. Given that there were a number of questionnaires needing completed at each visit, it was felt to be in the patients' best interests for a study researcher to help the patient complete the questionnaires. Although this is not how these questionnaires were validated, it proved to be a most useful decision as many patients found the help provided by the study researcher to be most useful. The researcher used many models of Standard international questionnaire such as Brief Pain inventory (BPI), which evaluate the pain in one to ten scale correspond with time duration. Short form McGill pain question (SFMPQ), consist of 15 description (11 sensor, 4 affective) the scale is from (0-4) in this study we used sensory component only lead Assessment of Neuropathic symptoms and sign (LANSS) which pain questionnaires is divided into section A complete by patient and section B complete by clinician

Each of this model correspond with pain response rate, Quality of life and toxicity.

Ninety-five patients were female and the median age (IQR) was 71.50 (35-55) years. The median survival from the time of trial registration was 93 days (CI 68-118). The Median (IQR) baseline BPI score was 57 (42.0-65.5). There are many methods to measure it, such as direct method, actuarial method and Kalban-Meier method. First measure by the Direct Method (appendix- vital status data sheet), it's the simplest way of summarizing patient survival, by calculate the percentage of patients alive at the end of a specified interval such as 12 weeks, for the 100 patients at risk for at 12 weeks indicates that 39 Patients were alive at last contact and 61 had died before week 12. Palliative radiotherapy play important role in management of pain, we can evaluation the benefit of radiotherapy in palliative cases by a set of international standards questionnaire by which the calibration of the result A major goal of palliative radiotherapy is control the symptoms by given the tumour dose the lethal dose with simple treatment planning and treatment. However, patient's symptoms such as pain, compression symptoms can be controlled by radiotherapy.

\section{Recommendations}

a. Implantation of stander palliative radiotherapy program in each oncology hospital play important role in success the RT treatment.

b. The time is important factor which effect in patient's response, time between the symptoms appearance and treat with first session.

c. The radiotherapy regime which describe the suitable field size with minimum number of fractions should be chosen.

d. Radiotherapy for pain controlled recommend because of high response and low side effect.

\section{Acknowledgment}

I am most of all grateful to the God, who made me able to fulfil this $\mathrm{PhD}$ thesis in spite of all problem and pressure. Profound thanks and gratitude to everyone who encouraged me to complete this thesis. I named my thesis to all breast cancer patients fighting and win, My parents, my husband and my kids whose prayers

and support has always contributed to my success. My supervisor professor Mohamed Alfadil and Dr Nahla gafer deserves all my gratitude as I could not have made it through without his invaluable support.

\section{References}

1. Janjan N, Lutz ST, Bedwinek JM, Hartsell WF, Pieters RS Jr, et al. (2009) Clinical trials and socioeconomic implication in the treatment of bone metastasis: a report from the American College of Radiology Appropriateness Criteria Expert Panel on Radiation Oncology. Journal of Palliative Medicine 12(5): 427-431.

2. Cleeland CS, Ryan KM (1994) Pain assessment: global use of the Brief Pain Inventory. ANNALS Academy of Medicine Singapore 23(2): 129138.

3. Portenoy RK, Payne D, Jacobsen P (1999) Breakthrough pain: characteristics and impact in patients with cancer pain. Department of Pain Medicine and Palliative Care 81(1-2): 129-134.

4. Melzack R (1975) The McGill Pain Questionnaire: major properties and scoring methods. Department of Pain Medicine and Palliative Care 1(3): 277-299.

5. Melzack R (1987) The short-form McGill Pain Questionnaire. Department of Pain Medicine and Palliative Care 30(2): 191-197.

6. Dworkin RH, Turk DC, Revicki DA, Harding G, Coyne KS, et al. (2009) Development and initial validation of an expanded and revised version of the Short-form McGill Pain Questionnaire (SF-MPQ-2). Pain 144(1-2): 35-42.

7. Bennett M (2001) The LANSS Pain Scale: the Leeds assessment of neuropathic symptoms and signs. Pain 92(1-2): 147-157.

8. Kaki AM, El-Yaski AZ, Youseif E (2005) Identifying neuropathic pain among patients with chronic low-back pain: use of the Leeds Assessment of Neuropathic Symptoms and Signs pain scale. Regional Anesthesia and Pain Medicine 30(5): 422-428. 
9. Aaronson NK, Ahmedzai S, Bergman B, Bullinger M, Cull A, et al. (1993) The European Organization for Research and Treatment of Cancer QLQ-C30: a quality-of-life instrument for use in international clinical trials in oncology. Journal of the National Cancer Institute 85(5): 365376.

10. Whelan TJ, Pignol JP, Levine MN, Mark N. Levine, et al. (2010) Long-term results of hypo fractionated radiation therapy for breast cancer. New England Journal of Medicine 362: 513-520.
11. Yin W, Cheb B, Yu Y, Koag FM (2008) The growth of radiation oncology in mainland china during the last 10 year. International Journal of Radiation Oncology, Biology, Physics 70(3): 795-798.

12. Kirova YM, Campana F, Savignoni A, Laki F, Muresan M, et al. (2009) Breast-conserving treatment in the elderly: long-term results of adjuvant hypo fractionated and norm fractionated radiotherapy. International Journal of Radiation Oncology, Biology \& Physics 75(1):76-81.
(C) (P) This work is licensed under Creative

To Submit Your Article Click Here: Submit Article

DOI: $10.32474 /$ OAJOM.2019.02.000147

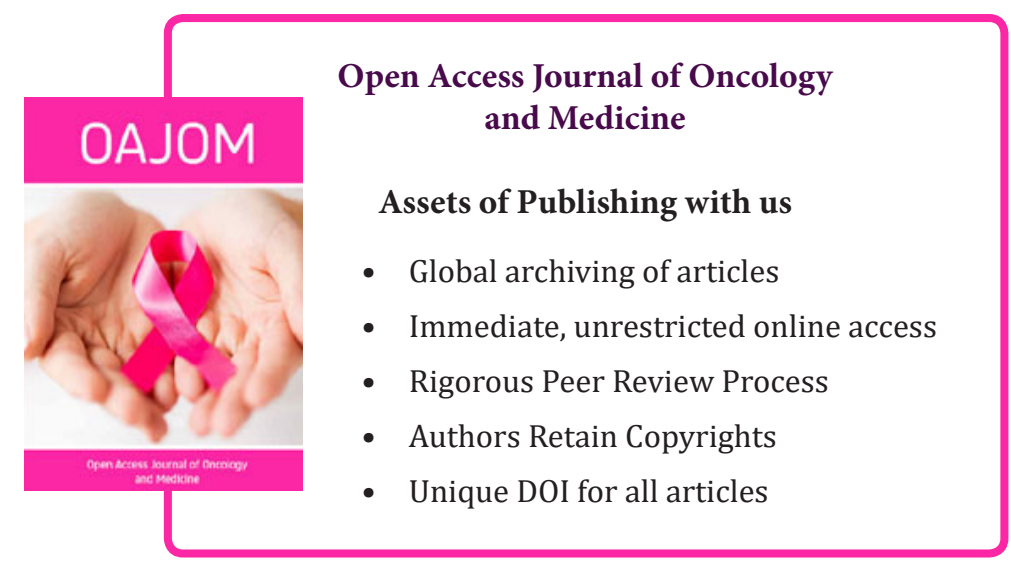

